\title{
[gw22-e0072] REDUCED EXPRESSION OF FXYD DOMAIN CONTAINING ION TRANSPORT REGULATOR 5 (FXYD5) IN ASSOCIATION WITH HYPERTENSION
}

Huang Xiaoyan', Shi Xaingxiang ${ }^{2}$, Hong Jian ${ }^{3}$, Zhou Xi', Geng Yong-jian ${ }^{4}$ Wenzhou Medical College; ${ }^{2}$ Wenzhou Medical Collegee, ${ }^{3}$ Baylor College of Medicine; ${ }^{4}$ University of Texas Health Science Center at Houston

10.1136/heartjnl-2011-300867.137

Background To investigate the expression of hypertensionrelated genes in spontaneous hypertension.

Methods A microarray screening for hypertension-related genes was conducted in rats with spontaneous hypertension (SHR) and rats with normotensive Wistar-Kyoto (WKY) using total RNA extracted from second-order mesenteric arteries and kidneys. Then the FXYD5 mRNA expression in vascular smooth muscle cells (VSMCs) was silenced by RNA interference (RNAi). Furthermore, the proliferation, mobility and $\mathrm{Na}+\mathrm{K}+$-ATPase activity of cell were analysed.

Results Among 10,000 genes examined, the expression of human gene homolog FXYD domain containing ion transport regulator 5 (FXYD5) was found 14.8-fold lower in SHR than that in WKY rats $(p<0.01)$. The result of microarray assay was confirmed using quantitative real-time RT-PCR and Northern Blot. The altered FXYD5 gene expression occurred in a timeand tissue-dependent manner. The FXYD5 gene expression was highest in SHR's kidney at age of 13 weeks when the blood pressure reached the highest levels. The downregulated FXYD5 inhibits the migration of smooth muscle cells $(\mathrm{p}<0.01)$ and cell membrane $\mathrm{Na}+\mathrm{K}+$-ATPase activity $(\mathrm{p}<0.01)$.

Conclusions The down-regulation of FXYD5 is related to the migration of smooth muscle cells and cell membrane $\mathrm{Na}+\mathrm{K}+-$ ATPase activity in rodents. So this gene may have profound impact on the regulation of blood pressure, and therefore is likely to act as a potential target for anti-hypertensive therapy. 